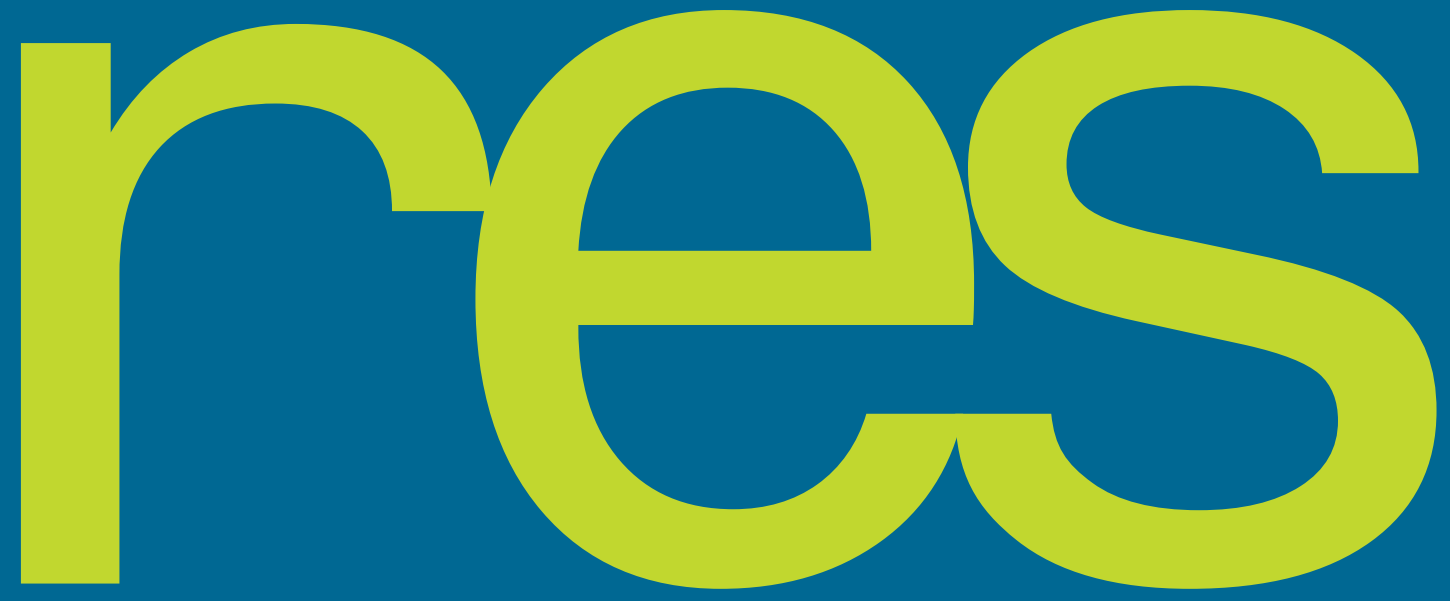

vol.12 • issue 3 • December 2020

\begin{abstract}
An Intellectual of the church: Remembering Father André Scrima (1925-2000)

Ein Intellektueller der Kirche: Erinnerung an André Scrima (1925-2000)
\end{abstract}




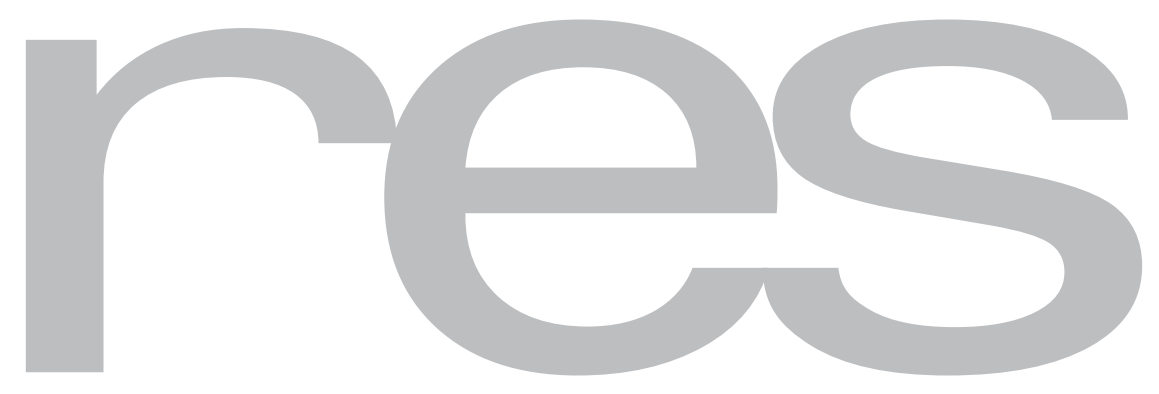

\section{Review of Ecumenical Studies • Sibiu}

$$
\text { vol. } 12 \text { issue } 3 \cdot \text { December } 2020
$$

An Intellectual of the Church: Remembering Father André Scrima (1925-2000)

Ein Intellektueller der Kirche: Erinnerung an André Scrima (1925-2000) 


\section{RES • Review of Ecumenical Studies • Sibiu}

\section{Academic Board / Wissenschaftlicher Beirat}

HE Laurenţiu Streza, Metropolitan of Transylvania/Lucian Blaga University of Sibiu

Bischof ReInHart Guib, Evangelische Kirche A.B. in Rumänien

LUIGINO BRUNI, LUMSA University, Rome

Nicolas Cernokrak, Saint-Serge Institute of Orthodox Theology, Paris

PIERo Coda, Sophia University Institute, Loppiano/Florence

WaLTER DiETRICH, Universität Bern

Basilius Groen, Karl-Franzens-Universität Graz

IOAN ICĂ JR, Lucian Blaga University of Sibiu

Pantelis Kalaitzidis, Volos Academy for Theological Studies, Volos, Greece

Bischof em. Christoph Klein, Evangelische Kirche A.B. in Rumänien/ Lucian Blaga University of Sibiu

Paul Niedermaier, Romanian Academy

Hermann Pitters, Lucian Blaga University of Sibiu

SR ÉLIANe PoIrot ocd, Monastère de Saint-Rémy / Schitul Stânceni

ERICH Renhart, Karl-Franzens-Universität Graz

CHRISTOPH SchwÖBEL, Eberhard-Karls-Universität Tübingen

Dorothea Wendebourg, Humboldt-Universität zu Berlin 


\section{RES $\cdot$ Review of Ecumenical Studies $•$ Sibiu}

$12 \cdot 3 \cdot 2020$

\section{ConTENTs / InhaLT}

Editorial

\section{Articles / Aufsätze}

Anca Manolescu

André Scrima: Orality and Writing

Georgiana Huian

Einblicke in die apophatische und mystische Bedeutung

der theologischen Anthropologie von André Scrima

Dragoș BoIcu

Epiphany and Otherness in the Vision of Father André

Scrima

Mihai-IUlian Dancă

André Scrima, Reader of Heidegger: From the Desert of

Being to the Desert of the Monastery

IOAN Alexandru Tofan

Reflection on Mediation: Interpreting a Book of Andrei

Scrima

Bogdan TĂTARU-CAZABAN

485

The Inner Dimension of the Orthodox Tradition and

Traditionalism According to André Scrima's Hermeneutics

Iuliu-Marius Morariu

497

Elements of Father Andrei Scrima's Ecumenical Activity as Reflected in File No. 0005468 from the "Securitate" Archives

Marius VAsIlEANU

Father André Scrima - A New Kind of Fool for Christ 


\section{Book Reviews / Buchrezensionen}

André Scrima, L'évangile de Jean. Un commentaire, trans. Marcel Pirard, ed. and introd. Anca Vasiliu, Paris, Cerf 2017 (Antoaneta Sabău). 526

André Scrima, L'accompagnamento spirituale. Il movimento del Roveto ardente e la rinascita esicasta in Romania, ed. and trans. Adalberto Mainardi, Spiritualità Orientale, Magnano, Edizioni Qiqajon 2018 (Alexandru Marius Crișan)

André Scrima, Apophatic Anthropology. An English Translation, trans.

Octavian Gabor, Perspectives on Philosophy and Religious Thought 17, Piscataway, New Jersey, Gorgias Press 2016 (Florin George Calian)

Daniela Dumbravă et Bogdan Tătaru-Cazaban (textes édités par), André Scrima. Expérience spirituelle et langage théologique. Actes du colloque de Rome, 29-30 octobre 2008, Orientalia Christiana Analecta 306, Roma, Pontificio Istituto Orientale 2019 (Ionuț Biliuță)

Cristian Sibișan, Theanthropologie - Grundzüge der Anthropologie und Christologie André Scrimas, Hamburg, Verlag Dr. Kovač 2017 (Marian Pătru)

Hans Bruno Fröhlich, Eine einmalige Gabe. Die Frage der Anerkennung der christlichen Taufe zwischen evangelischer und orthodoxer Kirche und die Praxis in Rumänien, Sibiu/Hermannstadt, Honterus-Verlag 2020 (Cosmin Pricop)

Simone Sinn, Dina El Omani und Anne Hege Grung (Hg.), Heilige Schriften heute verstehen. Christen und Muslime im Dialog, LWBDokumentation 62, Leipzig, Evangelische Verlagsanstalt 2019 (Hermann Dankwart Pitters)

Panayiotis Tzamalikos, Anaxagoras, Origen, and Neoplatonism. The Legacy of Anaxagoras to Classical and Late Antiquity, Vol. I and II, Arbeiten zur Kirchengeschichte 128/I and 128/II, Berlin-Boston, De Gruyter 2016 (Florin George Calian)

\section{André Scrima at the Institute for Ecumenical Research/ André Scrima am Institut für Ökumenische Forschung}

Mystical Theology in the Muslim-Christian Dialogue: Chiara Lubich, André Scrima and Classical Persian and Arab Authors. Hasso-Plattner Funded Research Project (Antoaneta Sabău) 


\title{
Einblicke in die apophatische und mystische Bedeutung der theologischen Anthropologie von André Scrima*
}

\author{
GEORgIANA HUIAN ${ }^{* *}$
}

This article investigates two fundamental dimensions of André Scrima's anthropology: his emphasis on the incomprehensibility of the human being, and his interest for the mystical life in spiritual experience. The author intertwines these aspects in a range of topics with the aim of approaching the nature of the human being, such as the access to God as presence, the deification or transfiguration of the human being, and the iconic character of human existence. I analyze the use of such terms like "participation" and "mixture," as well as the imagery that depicts the union of the human and the divine. Finally, I underline the spiritual importance of the nomad as figure and hospitality as virtue, and interpret them in terms of human itinerancy and God's mystical dwelling in the human person.

Keywords: anthropology, apophaticism, transfiguration, deification, image of God, mystery, participation

\section{Einführung}

Der Lebensweg André Scrimas (1925-2000) ${ }^{1}$ ähnelt dem Weg einer intellektuellen, geistlichen, akademischen und ökumenischen Pilgerschaft. Nach lebhaftem Interesse für Medizin, Philosophie und mathematisch-physikalische

\footnotetext{
* Für ihre Empfehlungen angesichts der Systematisierung und Vertiefung der Problematik sowie für konkrete Verbesserungsvorschläge danke ich den Gutachtern des Aufsatzes für die Review of Ecumenical Studies und den Mitgliedern des Forschungsprojekts „Image of God and Abyss of Desires“ (Bern): Rainer Hirsch-Luipold, Beatrice Wyss, Athanasios Despotis und Ilya Kaplan. Für die Zurverfügungstellung von verschiedenen neuesten Aufsätzen über A. Scrima bin ich Frau Anca Vasiliu besonders dankbar.

** Georgiana Huian, Assistenzprofessorin für Systematische Theologie und Ökumene, Institut für Christkatholische Theologie, Theologische Fakultät der Universität Bern, Längassstrasse 51,3012 Bern, Schweiz, georgiana.huian@theol.unibe.ch, georgiana.huian@ yahoo.com.

${ }^{1}$ Für biographische Informationen siehe: Anca Vasiliu, „André Scrima, l'étranger“, Contacts 56, Nr. 207 (2004): 211-23; Olivier Clément, „Note Biographique“, Contacts 55, Nr. 203 (2003): 243-45; Père Élie de Deir-el-Harf, „Témoignage,“ Contacts 55, Nr. 203 (2003): 246-62; Sœur Noëlle Devilliers, „Ce que fut dans ma vie la rencontre du père André Scrima,“ Contacts 55, Nr. 203 (2003): 263-72; Cristian Sibişan, Theanthropologie - Grundzüge der Anthropologie und Christologie André Scrimas (Hamburg: Verlag Dr. Kovač, 2017), 11-28: Kurze Biographie André Scrimas. Ein Dossier zu André Scrima mit Fotos und Zeugnissen wurde in Dilema veche 17, Nr. 854 (20.-26. August 2020) veröffentlicht.
} 
Wissenschaften lernt der junge Scrima die hesychastisch-geprägte Bewegung „Der Brennende Dornbusch“ kennen und fängt an, Theologie in Bukarest zu studieren. 1949 wurde Scrima Novize der monastischen Gemeinde Antim in Bukarest; 1956 wurde er im Kloster Slatina zum Mönch geweiht. Im gleichen Jahr hat er sein Theologiestudium mit einer Magisterarbeit zur apophatischen Anthropologie aus der Sicht der orthodoxen Tradition unter der Betreuung von Prof. Dumitru Stăniloae abgeschlossen. Auslandsreisen führen Scrima nach 1956 in die Schweiz, nach Indien, in den Libanon und nach Paris. Als ökumenische Figur wurde er Vertreter des Ökumenischen Patriarchen Athenagoras beim Zweiten Vatikanischen Konzil und hat das Treffen von Athenagoras und Paul VI. im Jahr 1964 vorbereitet. Sein akademisches Leben umfasst Unterrichtszeiten am Institut der Dominikaner Le Saulchoir (Paris, 1966-1968), an der Maronitischen Heilig-Geist-Universität (Kaslik, 1970-1974), an der Fakultät für Religionswissenschaft der SanktJoseph-Universität im Libanon (Beirut, 1974-1980) und eine Visiting Professorship an der Divinity School der Universität Chicago (auf Einladung von Mircea Eliade). Nach dem Zusammenbruch des Kommunismus entschloss sich Scrima, nach Rumänien zurückzukehren, wo er während etwa 10 Jahren seine intellektuelle Tätigkeit weiterführte mit einem besonderen Bezug zum New Europe College in Bukarest. Am New Europe College fand, nach der Beendung seiner irdischen Pilgerfahrt, auch das Archiv „André Scrima" seine Heimat.

Die theologische Anthropologie von André Scrima hat eine sehr enge Beziehung mit seinem Verständnis von Mystik und Spiritualität. Man könnte sogar sagen, dass sich zwischen der Sprache der dogmatischen Überlegungen und der Sprache der geistigen, liturgischen und mystischen Erfahrung ein enges Verhältnis entwickelt: eine gegenseitige Durchdringung, die die beiden Ausdrucksformen zu einem untrennbaren Ganzen vereint, aber ihre jeweilige Identität bewahrt. In der folgenden Untersuchung werden einige Elemente der theologischen Anthropologie Scrimas hinsichtlich ihrer apophatischen Ansprüche analysiert und evaluiert. ${ }^{2}$ Die Auswahl der Elemente erfolgt in einem Versuch der Systematisierung von Scrimas Denken, der zugleich seine dogmatischen Kernpunkte, seine patristische Verwurzelung, aber auch das Bruchstückhafte und Blitzhafte seiner Denksequenzen - die auf geistige Intuition aufbauen - sichtbar machen möchte. Somit wid-

\footnotetext{
2 „Among the Eastern Orthodox, André Scrima has perhaps accomplished the most thorough-going and explicit Patristic and Orthodox demonstration of the apophatic aspect of anthropology..." in Joshua Schooping, „Apophatic Anthropology and Hesychasm: Attending to the Mysterious Depths of Human Being", International Journal of Orthodox Theology 10, Nr. 4 (2019): 49-85, hier 52-53.
} 
met sich die Analyse folgenden Themen: die Wirkung von Gottes Licht und vom Heiligen Geist im menschlichen Wesen; das Verstehen der Vergöttlichung unter der Kategorie der „Anwesenheit“ und der göttlichen Assimilation; der Mensch in seiner ikonischen Identität und Dynamik; die Neuschöpfung des Menschen in Christus; die Körperlichkeit und die Beziehung des Menschen zur Materialität und Zeitlichkeit; die Freiheit und die Beziehung zum Mysterium; der Mensch als Wanderer und die Berufung der Gastfreundschaft. Im Licht der Analyse dieser Themenbereiche versuche ich gleichzeitig zu zeigen, dass die Anwendung der apophatischen Methode in der Anthropologie eine geistige Erfahrung und eine Auffassung von Mystik zum Ausdruck bringt. Die Untersuchung befasst sich hauptsächlich mit einer Auswahl von Ideen, die in den Bänden Die apophatische Anthropologie (Antropologia apofatică, 2005) und Die spirituelle Erfahrung und ihre Ausdrucksformen (Experienţa spirituală şi limbajele ei, 2008) vorkommen.

\section{Hesychastische Erfahrung im Licht Gottes und im Heiligen Geist}

Da Scrimas theologisches Denken von der hesychastischen Tradition geprägt ist, bietet es sich an, die Darstellung der negativen Anthropologie mit den Betrachtungen zur menschlichen Erfahrung der Teilhabe an den ungeschaffenen Energien Gottes zu beginnen. Der Apophatismus zeigt sich durch die Teilhabe des geschaffenen Wesens an dem göttlichen Licht, und dieses geschieht durch die Anwesenheit des Heiligen Geistes in der menschlichen Seele. Weder das göttliche Licht noch die Präsenz des Heiligen Geistes können durch positive Bestimmungen erfasst und durch sprachliche Mittel umfassend ausgedrückt werden. Weil der Mensch sich danach sehnt, tief und innig im „Mysterium der Anwesenheit des Geistes“3 geborgen zu sein, braucht er ebenfalls eine apophatische Herangehensweise. Für A. Scrima ist „Apophatismus“ nicht nur eine Dimension der Anthropologie, sondern ein „Zeugen“ für die Wirkung des Heiligen Geistes ${ }^{4}$ im Menschen und in der Schöpfung überhaupt.

Durch das unaussprechbare Leben vom Geiste beschränkt sich das menschliche Wesen nicht nur auf dessen Erkenntnis, sondern erfüllt tatsächlich auch die erste und die wahre Selbsterkenntnis. Im unerschaffenen Licht, das in seinem Inneren glänzt, ent-

\footnotetext{
${ }^{3}$ André Scrima, Antropologia apofatică [Die apophatische Anthropologie], hg. von Vlad Alexandrescu (Bukarest: Humanitas, 2005), 140 (im Folgenden durch $A A$ abgekürzt; alle Übersetzungen von Scrimas Schriften und Manuskripten stammen von mir).

${ }^{4}$ Ibidem, 140.
} 
deckt das Geschöpf auch seine eigenen Tiefen: das tiefe personale Bewusstsein (nicht nur die einfache psychologische Reflexion) ist ontologisch gleichzeitig mit dem noch tieferen Bewusstsein der Anwesenheit der Person des Geistes. ${ }^{5}$

Die apophatischen Eigenschaften und Aussagen, die dem Menschen zukommen, sind aus dem theologischen Apophatismus, der nur Gott als unerschaffenem Wesen zukommt, abgeleitet. Für Scrima steht die Teilhabe des Menschen an den apophatischen Bestimmungen des Unerschaffenen nicht im Bereich des Übernatürlichen, sondern sie drückt das „Natürliche“ aus ${ }^{6}$. Nichts ist natürlicher, als dass der Mensch sich schließlich als eine „unaussprechbare“, unbegreifliche, „paradoxale“ und „antinomische Existenz" versteht $^{7}$. Dies bedeutet für Scrima nicht, sich ausschließlich auf die menschliche Existenz zu fokussieren - das wäre Idololatrie -, sondern die Wirkung des Heiligen Geistes in ihren letzten Konsequenzen zu betrachten.

Die spezifische Tätigkeit des Heiligen Geistes auf das menschliche

Geschöpf unterstreicht deutlich den impliziten und natürlichen Apophatismus des vergöttlichten Menschen. ${ }^{8}$

Einerseits, argumentiert Scrima, nur wenn man bei der Wirkung des Geistes in den "Zuständen der gnadenvollen Vollkommenheit“ beginnt, erschließen sich „der Wert und der Zielpunkt des menschlichen Wesens" ". Andererseits wird jeder Versuch eines diskursiv-intellektuellen Verständnisses des Mysteriums des Menschen unvermeidlich in der „instinkthaften Unbegreiflichkeit für die Ordnung des Apophatischen“ enden. Auf diese Weise werden die apophatischen Beschreibungen des Menschen nur als „vage Dichtung“ und „bloße Metapher“ betrachtet. Die Einordnung solcher Beschreibungen in den Bereich der Metaphorik und des Figurativen erschwert, ja macht sogar den Zugang zum „realen Bild des zur Teilhabe am lebendigen Gott völlig zugelassenen Geschöpfes" ${ }^{\text {"10 }}$ unmöglich.

\footnotetext{
${ }^{5}$ Ibidem.

${ }^{6}$ Ibidem.

${ }^{8} A A, 141$.

${ }^{9}$ Ibidem.

${ }^{10}$ Ibidem.
}

7 Ibidem, 140-41. Dass Termini wie „Unfassbarkeit“, „Unaussprechlichkeit“, „Unerkennbarkeit“, „Undenkbarkeit“ dem Apophatismus „eigen“ sind, unterstreicht auch die Analyse von Sibişan, Theanthropologie, 105. Für die „apophatische Antinomie“, ibidem, 87: „Die apophatische Antinomie steht in einer unabdingbaren Relation mit dem Apophatismus selbst, noch mehr: Sie ist als das Proprium oder als Hauptinhalt der apophatischen Theologie zu betrachten." Dementsprechend werden die Antinomie und das Antinomische auch eine wichtige Rolle im anthropologischen Apophatismus spielen. 


\section{Vergöttlichung: Gott als Anwesenheit}

Die hesychastische Erfahrung, die den Menschen zu seiner Vollkommenheit im Heiligen Geist erhebt, eröffnet die Rede über die Vergöttlichung des Menschen. Für André Scrima ist die Kategorie der Anwesenheit ein wichtiger Begriff für die Erklärung des Lebens im Heiligen Geist und der Vergöttlichung. Die „Anwesenheit“ erläutert auch die innerliche und verklärende Präsenz des "göttlichen Bildes des Geschöpfes"11. Die Anwesenheit hat eine ultimative und abgründige Bedeutung: „eine Präsenz ist all das, was als Innerliches und Tiefstes in der Seele existiert "12. Gott kann nur unter der species der Präsenz angeeignet werden - nichtsdestotrotz bleibt er in dieser Aneignung ,unaussprechbar und unerkennbar" - er ist aber auch wahrhaft „kommuniziert" „jenseits der Intelligenz in einer bestimmten, lebendigen und persönlichen Weise ". ${ }^{13}$ Die Anwesenheit ist also eine theologische Kategorie, die Gott als erfahrbar und kommunizierbar, aber nicht als erkennbar, erfassbar, begreifbar verstehen lässt. Sie hat in diesem Sinne gleichzeitig eine apophatische und eine mystische Dimension. Die Kategorie der Anwesenheit antwortet auf die Frage: Wie ist Gott zugänglich und wie erhält der Mensch Anteil an ihm? Der apophatisch-mystische Sinn wäre unter die "theonome Bedeutung “ ${ }^{114} \mathrm{der}$ „Anwesenheit" unterzuordnen. Deswegen hat die Kategorie der Anwesenheit immer auch eine „wesentlich anthropologische Funktion“, denn „sie konstituiert sich als dynamisches Zentrum der Strahlungen der heilenden Kräfte “. ${ }^{15}$

Als ein solches Zentrum kann die Anwesenheit Gottes im Menschen mit Scrimas Auffassung über die Vergöttlichung in Verbindung gebracht werden. Die Vergöttlichung des Menschen ist aufgrund einer „tiefen metaphysischen Kongruenz zwischen dem Menschen und Gott "16 möglich, die in der Erschaffung des Menschen nach dem Bild Gottes ihren anthropologischen Ausdruck bekommt. Sie wird aber verwirklicht und erfüllt durch die „göttliche Kindesannahme in Jesus Christus, als Frucht seiner Kenose“"17. In diesem Zustand begegnen sich die „Ökonomie des Sohnes“ mit der „Ökonomie des Heiligen Geistes“18: die Gnade des Geistes erlaubt es, dass

\footnotetext{
${ }^{11}$ Ibidem, 123.

${ }^{12}$ Ibidem.

${ }^{13}$ Ibidem.

${ }^{14}$ Ibidem.

${ }^{15}$ Ibidem.

${ }^{16}$ Ibidem, 126. A. Scrima erwähnt hier auch den Begriff der „,ewigen Theanthropie“, den er von Sergej Bulgakovs Schriften Du Verbe incarné (Paris: Aubier, 1946) und Le Paraclet (Paris: Aubier, 1946) übernimmt.
}

${ }^{17} \mathrm{~A} A, 126$.

${ }^{18}$ Ibidem, 127. 
die erste Präsenz Gottes, die vom Moment der Schöpfung ad imaginem Dei, mit der zweiten Präsenz, die vom Moment des Werdens zum Kind Gottes in Christus, sich auf tiefste Weise miteinander vereinen. ${ }^{19}$ Diese Vereinigung der zwei Anwesenheiten ereignet sich in dem, was Scrima den „gnadenvollen Zustand" (starea harică) des Menschen nennt: ein Zustand, in dem die Vergöttlichung des Menschen durch die vergöttlichende Gnade bewirkt wird. Diese Anwesenheit der „im eigentlichen Sinne vergöttlichenden Gnade“ kommt nicht mehr auf natürliche Weise jedem Menschen zu, der auf der Welt ist, sondern - historisch betrachtet - der „ersten Frucht der Menschheit, dem im mystischen Leib Christi zusammengesetzten Christentum “20. Aber durch die Menschwerdung Christi wird das menschliche Wesen potentiell Christus ähnlich gemacht (cristifiată); das bedeutet, dass die „lebenspendende Partizipation Gottes“ allen „ohne Einschränkungen oder Hindernisse“ eröffnet wird. ${ }^{21}$

Wichtig wäre hier noch zu bemerken, dass der Zustand der Vergöttlichung einen apophatischen Ansatz sowohl durch ihre christologischals auch durch ihre pneumatologisch-bedingten Dimensionen verlangt:

Auf der Ebene dieses Aktes und auf den Koordinaten des neuen ontologischen Regimes, das sich für das durch die Gnade vergöttlichte Geschöpf ergeben hat, empfängt der anthropologische Apophatismus eine neue Prägung und eine neue Begründung aus der Begegnung mit dem Apophatismus, der den zwei Personen der Trinität eigen ist: dem Gott-dem Sohn und dem Gott-dem Heiligen Geist. ${ }^{22}$

\section{Transfiguration und göttliche Assimilation}

Durch die Anwesenheit Gottes im Menschen wird in ihm eine Transformation, eine Transfiguration oder eine Verklärung bewirkt. Dass Gott ständig und wesentlich im Menschen präsent ist, ist nicht nur ein Ideal für den Menschen, sondern schlechthin der Modus Gottes „alles in allem“ (1. Kor 15,28) zu sein. Die Anwesenheit verwickelt oder zieht das Geschöpf in eine „neue und sublime Existenz": Gott transfiguriert das Geschöpf und „assimiliert“ es sich selber, und die „Assimilation“ zeigt sich darin, dass „der Schöpfer - aktuell, und nicht nur virtuell (potentiell) - alles in allem wird“23. Das Leben,

\footnotetext{
${ }^{19}$ Ibidem, 126.

${ }^{20}$ Ibidem, 127.

${ }^{21}$ Ibidem.

${ }^{22}$ Ibidem.

${ }^{23}$ Ibidem, 124.
} 
das durch diese Anwesenheit, Transfiguration und Assimilation entsteht, ist durch seine „übernatürliche Essenz" und „Struktur“ „ein verborgenes und mysteriöses Leben, unzugänglich für Sicht- und Verständnisweisen von außen“. ${ }^{24}$ Die Struktur dieses Lebens setzt ein verborgenes Zentrum voraus. Durch dieses Leben (und durch dieses Zentrum) kann man „in die transintelligible (aber nicht unintelligible) Ordnung des Apophatischen eintreten“25. Das Präfix „Trans-“ funktioniert für Scrima wie das Hyper der areopagitischen Schriften ${ }^{26}$. Es ist das sprachliche Vehikel der Veränderung des Menschen, der Verklärung und Vergöttlichung. Diese Veränderung - also die Transfiguration - bedeutet zugleich eine Reinigung, eine Erleuchtung, und eine Restaurierung des Bildes Gottes im Menschen, weil dieses „göttliche Bild“ - betont Scrima - eine Verdunkelung, eine Verminderung und sogar eine Verfälschung wegen der „Katastrophe“ des Sündenfalls „in Adam und

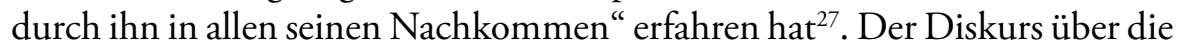
Vergöttlichung und über die göttliche Assimilation setzt also eine Diskussion zur Auffassung Scrimas über den Menschen als Bild Gottes voraus.

\section{Das Bild, die Ikone, das Ikonische}

Das Bild Gottes im Menschen ist eine „unverlierbare Wirklichkeit" ${ }^{\text {"28 }}$ der Mensch kann es nie auf „wirkliche und kategorische Weise“ verlieren, denn es macht seinen „ontologischen Kern“ aus. Wenn der Mensch dieses Bild verlieren würde, würde er „sein menschliches Wesen selbst verlieren,

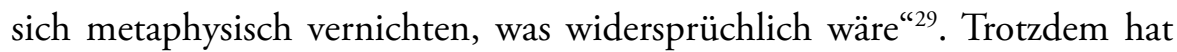
die „göttliche Ikone“ des menschlichen Wesens nicht ihren „edenischen Zustand der Reinheit und des ursprünglichen Glanzes“ behalten ${ }^{30}$. André Scrima betont, dass das Bild Gottes durch (oder nach) dem Sündenfall nicht verloren gegangen ist, dass es aber „bis zur Entstellung verändert [worden]“31 ist. Eine implizite Bedeutung der Schönheit und Harmonie scheint hier im Hintergrund zu stehen. Das Ziel des menschlichen Wesens ist es,

\footnotetext{
${ }^{24}$ Ibidem.

${ }^{25}$ Ibidem.

${ }^{27} A A, 124$.

${ }^{28}$ Ibidem.

${ }^{29}$ Ibidem.

${ }^{30}$ Ibidem.

${ }^{31}$ Ibidem.
}

${ }^{26}$ Sibişan, Theanthropologie, 75: „Kurzum: supra/hyper/über ist das direkte Korrelat des trans/jenseits - auf Rumänisch dincolo. Als wesenhaftes Proprium gehört diese Konstellation nur Gott." Durch Teilhabe an Gott kann diese Konstellation in abgeleitetem Sinne auch in dem anthropologischen Diskurs angewendet werden. 
in eine ikonische Beziehung mit seinem Schöpfer einzutreten, damit das Bild Gottes unverändert und vollkommen im Menschen strahlen kann und durch Schönheit, Glanz und Reinheit sichtbar und spürbar wird.

Es stellt sich die legitime Frage, worin genau das Bild Gottes im Menschen besteht: ob es in einer bestimmten Fakultät oder Eigenschaft zu finden oder ob es in einer Definition oder Bestimmung zu fassen ist, und ob diese Bestimmung nur relational, in einer Beziehung, die eine Dynamik der Ähnlichkeit voraussetzt, zu verstehen ist. André Scrima formuliert immer vorsichtig, um das Bild nicht in einer Was-Bestimmung zu definieren. Er versucht, die Konturen seiner Konzeption des Bildes nicht begrifflich $\mathrm{zu}$ begrenzen, damit sie permeabel für eine unendliche Beschreibung der Bildlichkeit in der menschlichen Schöpfung und in der Restaurierung in Christus bleiben. Wenn das Bild Gottes gerade das Unnahbare des unnahbaren Gottes im Menschen widerspielt, wenn es das Siegel des Werkes Christi und der Wirkung des Heiligen Geistes im Menschen empfängt und dadurch das Treffen mit dem christologischen und pneumatologischen Apophatismus vertieft, dann kann das Bild nur als abgründig und geheimnisvoll erscheinen.

André Scrima lokalisiert mehrmals das Bild Gottes in der Tiefe des Menschen oder in seinem geheimnisvollen Zentrum ${ }^{32}$, im Herzen ${ }^{33}$. Diese Situierung bedeutet jedoch nicht, dass dieses Bild mit dem Abgrund oder mit dem geheimnisvollen Herzen identisch ist, sondern eröffnet weitere Überlegungen, die um das Mysterium des Menschen kreisen. Diese Überlegungen haben aber die Beziehung zwischen dem deus absconditus und dem homo absconditus im Fokus ${ }^{34}$, welche als ikonische Beziehung das Mysterium des Menschen potenziert. Diese Beziehung beinhaltet die Bewegung, die Liebe und die freie Bewegung in Gott ${ }^{35}$. Sie fußt auf der im Menschen bestehenden „Fähigkeit, Gott zu leiden“ (pati Deum), oder anders gesagt, auf seinem Empfänglich-Sein für Gott (capax Dei) ${ }^{36}$ : der Mensch ist durch seine Gottebenbildlichkeit berufen, in eine pathische Beziehung mit

${ }^{32}$ Ibidem, 107. Scrima erklärt hier, dass "die göttliche Ikone“ in der "Gegend der Tiefe“ bleibt, „wo das innere mysteriöse Gesicht des Menschen sich verborgen befindet“. Im Einklang mit dem Augustinischen Entdecken Gottes als interior intimo meo (Confessiones $3,6,11)$, spricht er über den „,innersten, persönlichsten und dem Menschen am nächsten Kern", der eigentlich dem Anderen, also Gott, angehört.

${ }^{33} A A, 143$. Hier identifiziert Scrima die "letzte Tiefe in uns“ mit dem Begriff „cor absconditum".

${ }^{34}$ Ibidem, 98.

${ }^{35}$ Ibidem, 99: „...das [menschliche] Wesen existiert nur solange es in seinem unaussprechlichen ontologischen Zentrum anwesend ist, woraus es wahrhaft und auf tiefe Weise erkennen, lieben und sich in Gott bewegen kann."

${ }^{36}$ Ibidem, 115. 
Gott einzutreten. ${ }^{37}$ Das Bild Gottes ist der „innere Mensch“, der sich „durch Ähnlichkeit manifestiert" und unendlich nach dem Schönen strebt ${ }^{38}$. Es gibt also im Menschen eine unendliche Dynamik der Liebe, die als Objekt seiner Liebe die unendliche Liebe hat, die sich im Archetypus (in Gott) befindet ${ }^{39}$. Diese Dynamik impliziert, dass „das tiefe Bild des Menschen nie aufhören wird [...] sich unendlich auszudehnen, durch das Wachsen in der Ähnlichkeit, um den Ähnlichen zu empfangen “.40

Die Beziehung, die zwischen dem Bild und seinem Urbild entsteht und diese unendliche Dynamik voraussetzt, gehört zur Kategorie des Ikonischen. Das Ikonische ist daher nicht eine Bestimmung der Ikone als die eines Kultgegenstandes, der im ganzen liturgischen Leben der Ostkirche eine wichtige Rolle spielt, sondern es ist eine ontologische Situierung gegen-

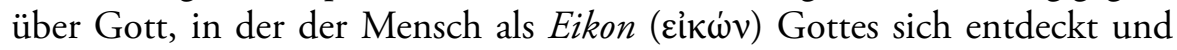
die Eikon durch das Wirken des Heiligen Geistes reinigen und immer strahlender erscheinen lässt. Diese ontologische Situierung könnte man auch als Anwesenheit Gottes im inneren Menschen erläutern, und die Läuterung dieses Bildes als Transfiguration. Sie hat zugleich epistemologische oder geistige Folgen (der Eintritt in das Transintelligible und das Apophatische) und mystische Konsequenzen (das Erfahren des Unerkennbaren, das Spüren des Unbegreiflichen, die Teilhabe des Geschaffenen am Unerschaffenen durch die unerschaffenen Energien Gottes, die aus dem verborgenen Zentrum des Menschen seine ganze menschliche Natur verklären). Das Ikonische ist für Scrima wesentlich, sowohl in der Charakterisierung der Heilsgeschichte als auch in der Beschreibung der Zeit vor der Menschwerdung Christi - wie im Negativ eines Fotos:

Zwischen dem ersten und dem zweiten Adam hat die Menschheit in einer an-ikonischen Epoche gelebt, in Ermangelung der Anwesenheit des tiefen Bildes und der inneren Anwesenheit Gottes. ${ }^{41}$

Diesen Satz könnte man als eine geschichtliche Aussage über den dramatischen Zustand der Menschheit vor der Rekapitulation und Restaurierung der menschlichen Natur in Christus lesen. Er spricht aber auch über die Bedingungen der ontologischen Vollkommenheit des Menschen und der Möglichkeit des mystischen Lebens: „das tiefe Bild“ und „die innere

\footnotetext{
${ }^{37}$ Auch die Vergöttlichung wird ,gelitten“ - sie ist daher die höchste Verwirklichung dieser pathischen Beziehung mit Gott, vgl. ibidem, 115.

${ }^{38}$ Ibidem, 116.

${ }^{39}$ Ibidem.

${ }^{40}$ Ibidem.

${ }^{41}$ Ibidem, 124.
} 
Anwesenheit Gottes“. Die Entdeckung des tiefen Bildes, der göttlichen Ikone, die im Menschen tief eingeprägt ist, ist der Ansatzpunkt der apophatischen Anthropologie. Die innere Anwesenheit Gottes eröffnet den Horizont für die geistliche und mystische Erfahrung, denn in ihr wirkt der Heilige Geist, der alles transfiguriert. Darüber hinaus wird hier unterstrichen, dass die Restaurierung des göttlichen Bildes vom menschlichen Wesen nur in Christus möglich wurde ${ }^{42}$. Nur in Christus also konnte das Bild Gottes zu seiner edenischen Schönheit zurückkehren, und darüber hinaus zu einer höheren Schönheit, die der vergöttlichten Menschen, erhoben werden.

\section{Der Neue Adam und der christusbezogene und christusförmige Mensch}

Die „Restaurierung“ des menschlichen Wesens ist „die Frucht einer Neuschöpfung, die vom wahrhaften Protovater (engl. protoparent) der Menschheit, dem Neuen Adam Jesus Christus, durchgeführt wird“ ${ }^{\text {“33. André }}$ Scrima überarbeitet hier die berühmte Typologie von Adam-Christus ${ }^{44}$ : die menschliche Natur ist berufen, christusbezogen - auch im Sinne von christusteilhaftig und so christusformig ${ }^{45}$ - zu werden, denn Christus ist der wirkliche Prototyp oder Archetyp der Menschheit. In ihm erhält die menschliche Natur, die „korrupt“ geworden ist, „ihre einstige Permeabilität dem Göttlichen gegenüber“. ${ }^{46}$ Der Begriff der „Permeabilität“ erscheint hier sehr wichtig; trotz-

\footnotetext{
${ }^{42}$ Ibidem, 125.
}

${ }^{43}$ Ibidem.

${ }^{44}$ Die biblischen Referenzen für diese Typologie sind: Röm 5,12-17; 1 Kor 15,45-49; und auch, bezüglich der Neuschöpfung des Menschen in Christus, 2 Kor 5,17. S. H. A. Lombard, „The Adam-Christ Typology in Romans 5:12-21,“ Neotestamentica 15 (1981): 69-100; Maja Weyermann, „Die Typologien von Adam-Christus und Eva-Maria und ihr Verhältnis zueinander," Internationale Kirchliche Zeitschrift 88, Nr. 2 (1998): 204-24; Otfried Hofius, „Die Adam-Christus Antithese und das Gesetz,“ in Paulusstudien II (Tübingen: Mohr Siebeck, 2002), 62-103; Marco Hofheinz, „Einleitung. Narrative Ethik als «Typfrage». Entwicklungen und Probleme eines konturierungsbedürftigen Programmbegriffes", in Ethik und Erzählung. Theologische und philosophische Beiträge zur narrativen Ethik, hg. von Marco Hofheinz, Frank Mathwig und Matthias Zeindler (Zürich: TVZ, 2009), 11-68 (über AdamChristus: 60-63). Zur Typologie: Karl-Heinrich Ostmeyer, „Typologie und Typos: Analyse eines schwierigen Verhältnisses“, New Testament Studies 46, Nr. 1 (2000): 112-31; idem, "Typos - weder Urbild noch Abbild," in Bilder-Sprache verstehen. Interdisziplinäre Studien zur Hermeneutik figurativen Sprechens, hg. von R. Zimmermann, mit einem Geleitwort von H.-G. Gadamer (München: Finck Verlag, 2000), 217-38.

${ }^{45}$ Hier steht „christusbezogen“" oder „christusförmig“ für das rumänische Adjektiv „ „ristic“ im Unterschied zu „christlich“ (rumänisch „creştin“). Für den Unterschied zwischen „,christusbezogen" und "christlich" in Scrimas Verständnis siehe Sibişan, Theanthropologie, 201.

${ }^{46} A A, 125$. 
dem wird er nicht weiter entfaltet oder erläutert ${ }^{47}$. Eine sehr wichtige patristische Quelle für diesen Begriff könnte die Auffassung von Gregor von Nazianz über das Menschsein bieten ${ }^{48}$. Bei Gregor ermöglicht die menschliche Permeabilität die Teilhabe am Leben Gottes; sie gründet ihrerseits in der ikonischen Beschaffenheit des Menschen ${ }^{49}$. Dieser Begriff könnte mit der Idee der „Plastizität ${ }^{\text {50 }}$ in Verbindung gebracht werden, auch wenn die beiden nicht völlig synonym sind. Der Mensch kann eine höhere Existenz erleben als das Leben, welches der „erste Adam“ vor dem Sündenfall erlebt hat:

${ }^{47}$ Es gibt wenig Forschung zur Permeabilität als theologischem Begriff, der ein ganzes Denkparadigma gründen könnte. Ein Beispiel: Joris Geldhof, „A paradigm of permeability: Franz von Baader on love", International Journal of Philosophy and Theology 78, Nr. 1-2 (2017): 91-105, DOI: 10.1080/21692327.2016.1246198, abgerufen am 20. Februar 2020. ${ }^{48}$ S. Carm. 1.2.18, PG 37, 787.13-14; Carm. 1.2.34, PG 37, 947.20; Or. 38.11 in Grégoire de Nazianze, Discours 38-41, hg. von Claudio Moreschini, übers. von Paul Gallay, SC 358 (Paris: Cerf, 1990), 124-26.

${ }^{49}$ S. Gabrielle Thomas, The Image of God in the Theology of Gregory of Nazianzus (Cambridge: Cambridge University Press, 2019): 70: „Therefore, it is possible for human persons to share in God's life due to their propensity for permeability. In Gregory's case, this permeability is possible for a human person precisely because she is made according to the eikon of God". G. Thomas zitiert in Bezug auf den Permeabilität-Gedanken einen Aufsatz von Paul Gavrilyuk, „The Retrieval of Deification. How a Once-Despised Archaism Became an Ecumenical Desideratum“, Modern Theology 25, Nr. 4 (2009): 647-59, hier 649. Sie bringt auch die Idee der „Permeabilität“ mit anderen modernen Begriffen wie „poröses Selbst“ („porous self“) oder „verwundbares Selbst“ („vulnerable self“) in Verbindung (The Image of God, 70). ${ }^{50}$ Siehe die Anwendungen des Begriffes in der neueren Auseinandersetzung mit dem Denken von Maximus Confessor: „Personhood reveals the "plasticity» of nature conducive to communion." - so Paul Blowers, Maximus the Confessor: Jesus Christ and the Transfiguration of the World (Oxford: Oxford University Press, 2016), 326, mit Bezugnahme auf Willis Jenkins, Ecologies of Grace. Environmental Ethics and Christian Theology (NY: Oxford University Press, 2008), 123-24. Der Begriff der „Plastizität“ kann auch die Transformationen der persönlichen Identität „in der Ich-Du Beziehung“ bezeichnen und spielt eine besondere Rolle im Denken der Relationalität bei Dumitru Stăniloae. Siehe: Marian Pătru, „Mutuality - Presence - Personal Revelation through the Word. Father Dumitru Stăniloae and Martin Buber's Relational Ontology“, Review of Ecumenical Studies 5, Nr. 3 (2013): 320-38, hier 331. Für Dumitru Stăniloae hat die „Plastizität“ eine allgemeinere Bedeutung in Bezug auf die ganze Schöpfung, denn die geschaffenen Dinge sind die „plastifizierten göttlichen Gedanken“ - Teologia dogmatică ortodoxă, Bd. 2 (Bucureşti: Editura Institutului Biblic şi de Misiune al Bisericii Ortodoxe Române, 2003), 7. Sie sind also logoi, die in der Ordnung des Sichtbaren Gestalt und Inhalt bekommen haben und „in die ontologische Dichte der konkreten Dinge transponiert“ worden sind. Doru Costache, „At the Crossroads of Contemporary Cosmology and the Patristic Worldview: Movement, Rationality and Purpose in Father Dumitru Stăniloae“, Studii Teologice 9, Nr. 2 (2013): 111-34, hier 122. Über das „Hinuntersteigen Gottes zu seiner Schöpfung“ als „Plastizität der göttlichen ratio“ bei Dumitru Stăniloae, siehe: Liviu Jitianu, „Eine theologische Anthropologie aus orthodoxer Sicht. Mystische Grundzüge der Menschenlehre im Werk von Dumitru Stăniloae“, Studia Universitatis Babeş-Bolyai 52, Nr. 2 (2007): 33-67, hier 48. 
Durch die Handlung des Erlösers wird der christusbezogene (christförmige) Mensch nun in Potenz vergöttlicht und kann, in diesem Leben sogar, den Zustand des nächsten Zeitalters in der vollkommenen Heiligung erreichen. ${ }^{51}$

Diese ganze Problematik der Restaurierung des Bildes Gottes und der Neuschöpfung des Menschen in Christus eröffnet die Frage nach der Art der Permeabilität für das Göttliche und nach der Beziehung zwischen den Ideen der Permeabilität (für), Teilhabe (an) und Mischung (mit). Wie können alle diese Begriffe und Bilder ihren Platz finden innerhalb einer anthropologischen Auffassung, die das Bild Gottes ins Zentrum setzt?

\section{Lebendiges und göttliches Bild. Zwischen Teilhabe ${ }^{52}$ und „Mischung“}

Was ist dieses Bild und wie ist es beschaffen, damit es die Vergöttlichung zulassen kann? A. Scrima warnt davor, dass man das Bild nicht auf eine künstlerische, malerische, mimetische Weise verstehen sollte, sondern auf eine lebendige und relationale Weise. Die Eikon ist weder ein Porträt noch eine Abbildung Gottes oder eine äußere und formelle imitatio. ${ }^{53}$ Das authentische Bild ist also unbedingt ein „lebendiges Bild“ und das „Organ der substantiellen Teilhabe am Leben der Gottheit“, deswegen kann es „die göttliche Ikone des Menschen“ genannt werden ${ }^{54}$. Dass das Bild selber göttlich ist und nicht nur das Abgebildete (das Göttliche), dass der menschlichen Eikon das Attribut des Göttlichen zukommt, ist eine Idee, die wieder stark an Gregor von Nazianz erinnert. ${ }^{55}$ Das Göttlichsein der Eikon erklärt sich dadurch, dass zwischen dem Bild und seinem Urbild eine Beziehung der gegenseitigen Angehörigkeit besteht. Die Betonung der Gegenseitigkeit und der Kohäsion ist ein wichtiger Zug der Anthropologie Scrimas:

[...] zwischen dem Bild und dem Archetyp gibt es eine

Angehörigkeit und eine gegenseitige Kohäsion. Dadurch ist das

\footnotetext{
${ }^{51} A A, 125$.

52 Über die Terminologie der „Teilhabe“, „Teilnahme“ oder „Anteil haben“ an Gott oder an seinem Leben, siehe: Sibişan, Theanthropologie, 75.

53 Schooping, „Apophatic Anthropology“, 60: „The Image of God is what constitutes and in-forms man's ontology, and as such "the general foundations of apophatic anthropology [are grasped] in this essential concept.». Man is a living icon, and so he is inwardly and ontologically directed towards his Archetype, not merely outwardly and ethically. At the depths, even at the metaphysical core of his being, man is theocentric".

${ }^{54} A A$, S. 122.

55 Thomas, The Image of God, 70: „The porous self signifies that the human person is so permeable or porous that when God breathes life into her, the eikon can be described as a "god" or «divine»" (...) „Gregory understands literally the divinity of the eikon, precisely because of her porosity to the Holy Spirit“.
} 
Geschöpf ab origine Gott geweiht und zugelassen zur wirklichen Teilhabe an Gottes Sein (divinae naturae consortes 2 Petr 1,4). ${ }^{56}$

Die Beziehung zwischen dem Bild und dem Urbild wird also zur Bedingung der Möglichkeit für die Teilhabe des geschaffenen Menschen am ungeschaffenen Gott. Ferner versucht André Scrima, die Modalitäten der Kohäsion und der Teilhabe zu verdeutlichen. Die „geistige Gemeinschaft mit Gott“ unterscheidet sich selbstverständlich wesentlich von einer „materiellen Vereinigung“: es geht hier nicht um eine „physische Verschmelzung der Substanzen, sondern um die zwei Prinzipien des Natürlichen und des Übernatürlichen. Sie sind miteinander «vermischt» und bilden ein organisches Ganzes, eine Natur höherer Ordnung, wie der Körper und die Seele, oder die Pflanze und das auf sie Aufgepfropfte. ${ }^{\text {" } 77}$

Es ist interessant, dass Scrima hier von der üblichen Sprache der Perichorese abweicht und die Idee der Mischung (in Anführungszeichen jedenfalls) einführt. Die anerkannte Terminologie der Perichorese ${ }^{58}-$ die trinitarisch die Einheit der drei Hypostasen in einem Wesen darstellt ${ }^{59}$, christologisch die Weise der Vereinigung der zwei Naturen in der einen Hypostase Christi erklärt ${ }^{60}$, und anthropologisch auf die Vereinigung des Menschlichen mit dem Göttlichen in der Vergöttlichung hinweist - vermeidet Verben wie „verschmelzen“, „vermischen“, oder „zerfließen“. In diesem Sinne dient der Terminus Perichoresis dazu, die gegenseitige Durchdringung von zwei

\footnotetext{
${ }^{56} A A, 122$.

${ }^{57}$ Ibidem.

${ }^{58}$ Peter Stemmer, „Perichorese. Zur Geschichte eines Begriffs“, Archiv für Begriffsgeschichte 27 (1983): 9-55; Jacques Fantino, „Circumincession,“ in Dictionnaire critique de théologie, hg. von Jean-Yves Lacoste (Paris: Presses Universitaires de France, 1998), 228. Für neuere und kritische Bewertungen des Modells der Perichorese siehe Markus Mühling, „Abschied von der Perichorese? Asymmetrische Reziprozität als Bedingung der Entzogenheit im Wesen Gottes“, in Entzogenheit in Gott. Beiträge zur Rede von der Verborgenheit der Trinität, hg. von Markus Mühling und M. Wendte (Utrecht: Ars Disputandi, 2005), 187-204.

${ }^{59}$ Johannes von Damaskus, De fide orthodoxa 1, 14, in Des heiligen Johannes von Damaskus genaue Darlegung des orthodoxen Glaubens, übers. Dionys Stiefenhofer, BKV 44 (München: Kösel, 1923), 42: „Die Personen weilen und wohnen ineinander. Denn sie sind unzertrennlich und gehen nicht auseinander, sie sind unvermischt ineinander, jedoch nicht so, dass sie verschmelzen oder zerfließen, sondern so, dass sie gegenseitig zusammenhängen. Denn der Sohn ist im Vater und im Geiste, und der Geist im Vater und im Sohne und der Vater im Sohne und im Geiste, ohne dass eine Zerfließung oder Verschmelzung oder Vermischung stattfände. Und es besteht Einheit und Identität in der Bewegung, denn die drei Personen haben nur eine Bewegung, eine Tätigkeit." (revidierte Übersetzung).

${ }^{60}$ Maximus Confessor, Disputatio cum Pyrrho, PG 91, 337 CD. Johannes von Damaskus, De fide orthodoxa 3,7, BKV 44, 131: „Wir sagen zwar, die Naturen des Herrn durchdringen sich gegenseitig, gleichwohl aber wissen wir, dass die Durchdringung von Seiten der göttlichen Natur geschehen ist."
} 
Naturen (oder „Prinzipien“, Scrimas Kontext folgend) ohne Verschmelzung und Vermischung zu bezeichnen, ohne damit irgendeine Trennung oder Teilung in dieses Verhältnis einzuführen.

Indem Scrima sich von physischen Vorstellungen abgrenzt, kommt er trotzdem zum Bild der Mischung als einem Bild mit höherem explanativem Wert. ${ }^{61}$ Dass die Mischung in diesem Zusammenhang ein Bild ist, deuten die Anführungszeichen an. Die Sprache stößt an ihre Grenzen, wenn der Mensch die Beziehung zwischen Gott dem Archetyp und seinem göttlichen Abbild im Menschen erklären möchte. Das Bild der Mischung und die Anführungszeichen bedeuten also ein Überschreiten der begrifflichen und referentiellen Sprache, also das Anwenden der apophatischen Sprache. Obwohl Scrima ein materielles Verständnis der Vereinigung zwischen den beiden Prinzipien - dem Natürlichen und dem Übernatürlichen - deutlich ablehnt, benutzt er am Ende trotzdem organische Analogien. Diese (anscheinend) widersprüchlichen Verhältnisse innerhalb Scrimas Argumentation gehören zur Aufnahme des Antinomischen in eine apophatische Methode der Entfaltung einer Problematik, die die Intelligibilität und die Vernunft übersteigt. Die organischen Analogien sind ihrerseits enigmatisch und nicht selbst-erklärend: was charakterisiert die Einheit von Körper und Seele, oder die Einheit, die eine Pflanze mit der auf ihr aufgepfropften anderen Art bildet? Auch wenn alle diese Bilder und Analogien die Vergöttlichung sprachlich nicht erschöpfend behandeln können, bleibt die Vergöttlichung laut André Scrima „der Apex der Erfüllung des Menschen“: ein Zustand wo „sein Anfang und sein Ende in eins zusammenfallen werden"62.

\section{Das sabbatische Wesen}

In diesem Teil meiner Darstellung wende ich mich den Anthropologischen Notizen (Notațiuni Antropologice) zu - einem Ideenjournal, das auch im Band Die apophatische Anthropologie veröffentlicht wurde ${ }^{63}$. Es handelt sich um einzelne Überlegungen zu verschiedenen Themen, die als kürzere oder

\footnotetext{
${ }^{61}$ Anca Vasiliu nennt auch die Mischung, mixis, und die figurale Ähnlichkeit (eikon, image hypostatique) als Möglichkeiten der Begegnung des Einsamen mit dem Einzigen, des Menschen mit Gott, d. h. der Begegnung monos pros monon, die die Fülle der eschatologischen Erkenntnis in sich trägt (vgl. 1 Kor 13,12). Siehe: Anca Vasiliu, „Solitude du principe et singularité de l'homme. Langage théologique et réflexion philosophique chez André Scrima", in André Scrima. Expérience spirituelle et langage théologique, Actes du colloque de Rome, 29-30 octobre 2008, Textes édités par Daniela Dumbravă et Bogdan TătaruCazaban, Orientalia Christiana Analecta 306 (Roma: Pontificio Istituto Orientale, 2019), 57-74, hier 73.

${ }^{62} A A, 122$.

${ }^{63}$ Notatiuni Antropologice, in AA, 177-371.
} 
längere Aphorismen (oder im Sinne der asketisch-mystischen Capita) verfasst wurden. Aus diesen punktuellen Überlegungen und kurzen Erörterungen werde ich verschiedene Themen auswählen, die die apophatischen und mystischen Dimensionen der theologischen Anthropologie Scrimas erhellen.

Vergöttlichung wird in einer Notiz als die sabbatische Stimmung verstanden, also in einem Zusammentreffen von Ruhe und Festlichkeit:

In der Tat, der Sabbat des Geschöpfes ist nichts anderes als der Zustand der Vergöttlichung: Gott hat sich von allen seinen Dingen und in allen seinen Dingen ausgeruht. Der Mensch trägt im Zentrum seines Wesens, im Herzen, Gott, der in ihm ruht, als ein Prinzip seiner Vergöttlichung. ${ }^{64}$

Einerseits scheint die Idee des Prinzips an die philosophische Sprache der antiken griechischen Philosophie zu erinnern ${ }^{65}$, andererseits aber zeugt die Vorstellung des Herzens als Zentrum, woraus das Prinzip strahlen und die ganze Existenz transfigurieren kann, vom hesychastischen Erbe ${ }^{66}$. Darüber hinaus steht diese sabbatische Vorstellung in Beziehung zum Frieden des Gebetes: der einzige Weg zum „inneren Himmel“ wie zum „ursprünglichen Eden“ führt durch den „springenden Frieden des Gebetes“67. Für Scrima ist eigentlich das "In-sich Spiegeln des Denkens“ ein innerer Frieden, der die Seele erleuchtet und nährt ${ }^{68}$. Außerdem ist die sabbatische Vorstellung auch mit Gedanken über die Schöpfung und das Endziel des Menschen verbunden: Scrima interpretiert Gen 1,25-26 in einer liturgischen Sprache und überbrückt danach den ursprünglichen Sabbat mit dem eschatologischen Sabbat.

\footnotetext{
${ }^{64}$ Ibidem, 178.

${ }^{65}$ Vasiliu, „Solitude du principe et singularité de l'homme“, 57-74. Vasiliu diskutiert die Modalitäten der Begegnungen zwischen der Einsamkeit des Menschen und die Einzigartigkeit des Prinzips und situiert das Begegnen in den Bereich des Sich-Offenbarenden, nicht des Apophatischen, z. B. auf S. 60: „La solitude n'est pas une forme d'ascèse pour André Scrima. Elle n'est pas non plus un reflet de l'apophatisme théologique (dionysien, et plus largement néoplatonicien), mais constitue, au contraire, une expression de l'apophantisme (du révélé) et une forme de manifester au mieux la proximité, voire l'unité structurelle entre Dieu et le monde." ${ }^{66}$ A. Scrima hat sich mit der Darstellung und Übermittlung der orthodoxen monastischen Tradition - mit ihrer hesychastischen und philokalischen Ausprägung - sehr viel beschäftigt, beispielsweise in den folgenden Schriften: „Le monachisme orthodoxe: histoire, traditions, spiritualité" (ein Text von 1961-1962), „Aperçus sur l'hésychasme,“ (Kurs gehalten an der theologischen Fakultät der Heilig-Geist-Universität, Kaslik, Libanon, 1970-1971), veröffentlicht in André Scrima, Despre isihasm [Über den Hesychasmus] (Bukarest: Humanitas 2003), 23-151. Ein früherer Text bezeugt die Erfahrung der Gebetsbewegung vom Kloster Antim von Bukarest: „L'avènement philocalique dans l'orthodoxie roumaine“, Istina 3-4 (1958): 295-328 und 443-75. Eine Synthese befindet sich im Band: Timpul Rugului aprins. Maestrul spiritual în tradiţia răsăriteană. [Die Zeit des Brennenden Dornbusches. Der geistliche Meister in der östlichen Tradition], 2. Auflage (Bukarest: Humanitas, 2000).

${ }^{67} A A$, S. 178.

${ }^{68}$ Ibidem, 177.
} 
Laut Scrimas Genesis-Exegese wurde der Mensch „in der Vesper des sechsten Tages geschaffen, in der Vesper des Freitags, welcher der Beginn des Samstags ist “69. Deswegen ist der Mensch „ein sabbatisches Geschöpf“, und unterscheidet sich von den anderen Geschöpfen, indem er „den festli-

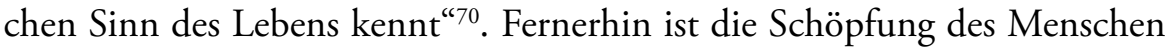
die Schwelle, das Tor, wodurch die ganze Schöpfung in den Frieden und in die Ruhe der Vereinigung mit ihrem Schöpfer übergeht. Der Sabbat ist also nicht nur das „Zeichen“ des Ins-Leben-Gerufen-Seins des Menschen, sondern auch sein „persönliches Schicksal“, seine Bestimmung und sein Ziel, seine „ontologische Modalität“, die zusätzlich eine christologische Begründung findet: „denn der Menschensohn ist Herr über den Sabbat“ (Mt 12,8; vgl. Mk 2,28; Lk 6,5). ${ }^{71}$

Der eschatologische Frieden, die „Ruhe des achten Äons“, anders gesagt „der herrliche Sabbat der Ewigkeit, die mit Gott verbracht wird“, kommen in Erfüllung aufgrund einer Kontinuität mit dem ursprünglichen Sabbat. Die Idee dieser Kontinuität verknüpft sich mit dem hesychastischen Ideal des Herabkommens des Reiches Gottes im Herzen des Menschen ${ }^{72}$, in dem der Kern seines sabbatischen Wesens geheimnisvoll bewahrt ist und sich im Gebet entfalten kann. Es ist hier bemerkenswert, dass die letzte Ruhe eine Dynamik voraussetzt, die in der festlich-ruhigen Beschaffenheit des menschlichen Herzens zu finden ist, wenn sie von der pneumatischen Ordnung durchdrungen und von der Herrlichkeit der Ausstrahlung Christi erhoben wird. „Das Anthropologische“ wird seine ontologische Funktion dann erfüllen, wenn die ganze Schöpfung - „im Menschen und durch den Menschen“ - ihren "Neueintritt in Gott und in die Sabbatruhe" verwirklichen wird. ${ }^{73}$ Diesen Neueintritt verwirklicht Christus als „Herr des Sabbats“. ${ }^{74}$

Das Verständnis des Menschen als sabbatisches Wesen unterstreicht die Verbindung zwischen dem Menschen und der ganzen Schöpfung, wobei sich die Frage der gemeinsamen Materialität stellen kann. In die-

${ }^{69}$ Ibidem. André Scrima benutzt das liturgische Wort „Vesper“ (vecernie), um die Zeit der Dämmerung des sechsten Tages zu bezeichnen, aber auch um den Vorabend und das Vorfeiern des Sabbats anzudeuten.

${ }^{70}$ Ibidem.

${ }^{71}$ Ibidem, 178.

72 Ibidem: „Das Reich der Himmel wird aus dem Herzen des Menschen kommen, worin es auf natürliche Weise hingesetzt ist: der objektive Plan und der subjektive Plan werden sich überwunden finden im pneumatischen Plan, in der Ordnung des Geistes, der die ganze Schöpfung aufwecken wird und in dessen Kraft der Sohn Gottes auf die Wolken des Himmels kommend zu sehen sein wird."

${ }^{73}$ Ibidem.

${ }^{74}$ Ibidem. 
sem Zusammenhang erhebt sich auch die Problematik der besonderen Stellung der menschlichen Leiblichkeit. Diese Leiblichkeit war die in der Fleischwerdung des Wortes Gottes, damit die ganze menschliche Natur erlöst und vergöttlicht werden kann.

\section{Körper und Kosmos}

Die Beziehung von Körper und Kosmos spielt eine wichtige Rolle in der Ordnung der Schöpfung und bezeichnet eine gewisse Kontinuität aufgrund der Materialität. Jedoch ist menschliche Körperlichkeit „permeabel“ zur Transfiguration in Christus, weil Christus das Wesen des Menschen ganz und gar in sich verklärt und vergöttlicht hat. Neben dieser christologischen Verankerung geht André Scrima in seinen Überlegungen auch einer asketischen Tradition nach, die die Rolle des Leibes in dem monastischen Leben reflektiert. In Hinblick auf eine Definition des Menschen als ,geistiges Wesen, das einen Leib trägt" (fiinţă spirituală purtătoare de trup), nennt Scrima die folgenden „großen Momente des Leibes“: „Wachen und Schlaf, Tod und Auferstehung, Transfiguration " ${ }^{75}$ Sicherlich ist es in dieser Aufzählung merkwürdig, dass alle diese Momente ausdrücklich als „Momente des Leibes“ identifiziert werden, wobei alle erwähnten Zustände sich nicht nur auf den Leib beziehen, sondern jeweils die enge Beziehung Leib-Seele charakterisieren. So sind z. B. Wachen und Schlaf Momente eines beseelten Leibes, oder genauer gesagt Momente eines lebendigen Wesens, dessen Seele in einem Körper lebt. Tod bedeutet das Verlassen des Leibes von der Seele, hingegen ist die Auferstehung als eine Wiedervereinigung der Seele mit dem Körper in einem höheren, durch den Geist durchdrungenen Zustand, zu fassen. Schließlich kann die Transfiguration auch nie den Leib allein betreffen, ohne die psychische und die geistige Dimension der menschlichen Existenz. Nichtsdestoweniger ordnet Scrima alle diese Zustände unter die Kategorie „Momente des Leibes“ - dadurch wird ersichtlich, dass Scrima im Begriff der Leiblichkeit immer eine (implizite) Bezogenheit auf die seelische und geistige Ebene voraussetzt.

Die „Transfiguration“ bildet nicht nur den Abschluss der Aufzählung, sondern steht für sich: sie gehört nicht zu einem Begriffspaar, sie ist in sich die Verwirklichung einer engen und paradoxalen Beziehung zwischen dem, was transfiguriert, und dem, was transfiguriert wird. In der Transfiguration ist die Beziehung Subjekt-Objekt, Aktiv-Passiv in einer engen Vereinigung sowohl vorausgesetzt als auch aufgehoben und übertroffen. Die Transfiguration, und im konkreteren Sinne die Verklärung oder Metamorphosis des Leibes durch den Geist, durch die unerschaffenen Energien Gottes, ist an sich eine

${ }^{75}$ Ibidem, 204. 
Verkörperung einer antinomischen Beziehung, die die Permeabilität des Leibes für den Geist und für das göttliche Leben ausdrückt.

Eine gewisse Transfiguration des Leibes geschieht in der monastischen Lebensweise, die mit dem Ausdruck „Engel im Leib“ gemäß der asketischen Tradition des Ostens wiederzugeben sei.

Für André Scrima ist diese antinomische aber transformationsreiche Bestimmung des „Engels im Leib“ „die Lebensweise des Menschen par excellence“, „die wahrhafte Lebensweise des Menschen“, die „den entscheidenden Ausgleich (echilibrul crucial) innerhalb des ontologischen Regimes des Menschen" ausführt. ${ }^{76}$

Wegen der Ambivalenz des Geistigen und des Materiellen, die der Mensch in sich trägt, und wegen der paradoxen und unerklärbaren Vereinigung Körper-Seele, wurde der Mensch in der patristischen Tradition als Mikrokosmos angesehen. Diese Tradition hat Wurzeln in Philon von Alexandria $^{77}$, entwickelt sich bei Origenes ${ }^{78}$, Basilius von Caesarea ${ }^{79}$, Gregor von Nazianz ${ }^{80}$, Gregor von Nyssa ${ }^{81}$, Gregor dem Großen ${ }^{82}$, Maximus

\section{${ }^{76}$ Ibidem.}

${ }^{77}$ Philon von Alexandria, Quis rerum divinarum heres sit 31.155. In Les oeuvres de Philon d'Alexandrie 15: Quis rerum divinarum heres sit, übers. von Marguerite Harl (Paris: Cerf, 1966), 240-43.

${ }^{78}$ Origenes, Contra Celsum 6.63. In Origenes, Contra Celsum - Gegen Celsus, eingeleitet und kommentiert von Michael Fiedrowicz, übers. von Claudia Barthold, Fontes Christiani 50/4 (Freiburg i. Br.: Herder, 2012), 1136-9. Hier wird auch das Problem diskutiert, ob die Wesensgestalt des Menschen „nach dem Bild Gottes“ sich in der Seele oder im Körper befindet.

${ }^{79}$ Basilius von Caesarea, Hexaemeron, Hom. 11.14 (PG 30, 55-58). In Basile de Césarée, Sur l'origine de l'homme (Hom. Xet XI de l'Hexaéméron), übers. von Alexis Smets und Michel Van Esbroeck, SC 160 (Paris: Cerf, 1970), 266-69 (Homélie 2, 14).

${ }^{80}$ Gregor von Nazianz, Oratio 38.11 (SC 358, 124-26), wo Gregor „depicts creation as brought to unity within the human microcosm“, laut Doru Costache, "Seeking Out the antecedents of the Maximian Theory of Everything: Saint Gregory the Theologian Oration 38", in: The Cappadocian Legacy: A Critical Appraisal, hg. von Doru Costache und Philip Kariatlis (Redfern, NSW: St Andrew's Orthodox Press, 2013), 225-42: hier 233. Siehe auch: Thomas, The Image of God, „Microcosm“, 71-72.

${ }^{81}$ Gregor von Nyssa, De hominis opificio 8 (PG 44, 144-49). Die „Legitimität der Analogie Mikrokosmos-Makrokosmos" ist auch in De anima et Resurrectione, 44B-C vorausgesetzt, laut Johannes Zachhuber, „The Soul as Dynamis in Gregory of Nyssa's On the Soul and Ressurection“", in Exploring Gregory of Nyssa: Philosophical, Theological, and Historical Studies, hg. von Anna Marmodoro und Neil B. McLynn (Oxford: Oxford University Press, 2018), 142-59, hier 149. Vgl. dazu Eugenio Corsini, „Lharmonie du monde et l'homme microcosme dans le Hominis Opificio", in Epektasis. Mélanges patristiques offerts au Cardinal Jean Daniélou, hg. von Jaques Fontaine und Charles Kannengiesser (Paris: Beauchesne, 1972), 455-62.

${ }^{82}$ Gregor der Große, Moralia in Iob 6.6.20 (CCL 143, 298): Uniuersitatis autem nomine homo signatur quia in ipso uera species et magna communio uniuersitatis ostenditur. Vgl. Ambrosius, 
Confessor $^{83}$ und strahlt aus bis zur hesychastischen Tradition und deren Synthese in Gregor Palamas ${ }^{84}$. André Scrima wird die Beziehung Mensch/ Kosmos umkehren und im Menschen nicht die Spiegelung in nuce des Kosmos sehen, sondern die integrierende Entität des großen Kosmos, mit anderen Worten, der ganzen Schöpfung. Somit wird der Mensch nicht mehr zu einem Mikrokosmos, sondern zu einem Makrokosmos. Dieser Gedankengang kehrt die erwartete Beziehung Mikrokosmos-Makrokosmos ${ }^{85}$, die auch philosophische Echos mitbringt, um.

Der Leib verleiht dem Menschen seine spezifische Bestimmung und Stellung; er verbindet ihn direkt mit der substantiellen Wirklichkeit des Kosmos und erlaubt ihm, ihn [den Kosmos] zu integrieren, sodass er [der Mensch] nicht ein Mikrokosmos, sondern ein Makrokosmos wird. Die schöpferische, antiphonische und dialektische Beziehung zwischen dem Geist und dem Leib manifestiert sich besonders in den Knotenpunkten des leiblichen Lebens, in seinen großen Momenten. „Der Engel im Leib“, das monastische Leben, ist das Einzige, welches sie erfasst und positiv auflöst. ${ }^{86}$

Die Überlegung Scrimas erinnert hier an den Gedanken des Maximos, dass der Mensch einen „synthetischen“ Charakter und also eine „vermit-

Hexaemeron 6.9.55, in Des heiligen Kirchenlehrers Ambrosius von Mailand Exameron, übers. von Joh. Ev. Niederhuber, BKV 17 (Kempten: Kösel, 1914), 278-79. Siehe für Kommentar: Carole Straw, Gregory the Great: Perfection in Imperfection (Berkeley - Los Angeles - London: University of California Press, 1988), 39.

${ }^{83}$ Lars Thunberg, Microcosm and Mediator: The Theological Anthropology of Maximus the Confessor (Lund, Copenhagen: Gleerup, Munksgaard 1965).

${ }^{84}$ Siehe: Gregor Palamas, Hom. 26, in The Homilies of Saint Gregory Palamas, übers. von Christopher Veniamin (South Canaan: Saint Tikhon's Seminary Press, 2004), 46-54, für den synthetischen und mikrokosmischen Charakter des Menschen. Vgl. Natural Chapters, über die vermittelnde Rolle des Menschen in der Schöpfung. Apud Demetrios Harper, „The Exemplar of Consubstantiality. St Gregory Palamas's Hesychast as an Expression of a Microcosmic Approach to Personhood“, in Personhood in the Byzantine Christian Tradition: Early, Medieval, and Modern Perspectives, hg. von Alexis Torrance und Symeon Paschalidis (New York: Routledge, 2018), 103-12.

${ }^{85}$ Für die Betrachtung dieser Beziehung, die bei Posidonius (durch Galen), Philo, Origenes, Gregor von Nyssa und Augustinus stark betont wird, siehe: Gerhard B. Ladner, „The Philosophical Anthropology of Saint Gregory pf Nyssa", Dumbarton Oaks Papers 12 (1958): 61-94, hier 71, Fußnote 47. Vgl. George P. Conger, Theories of Macrocosms and Microcosms in the History of Philosophy (New York: Columbia University Press, 1950); Rudolf Allers, „Microcosm from Anaximander to Paracelsus“, Traditio 2 (1944): 319-407; Marie-Thérèse d'Alverny, „L'homme comme symbole. Le microcosme“, in Simbol e Simbologia nell' alto medievo, Bd. 1 (Spoleto: Centro Italiano di Studi sull'Alto Medievo, 1976), 123-83; apud Straw, Gregory the Great, 39, Fußnote 47.

${ }^{86} A A, 204$. 
telnde" Rolle im Kosmos hat, da er die $\sigma \chi \varepsilon \dot{\varepsilon} \sigma 1 \varsigma^{87}$ zwischen dem Seelischen und dem Physischen darstellt ${ }^{88}$. Maximus' Ansicht einer „inneren Cosubstantialität ${ }^{\text {"89, }}$, die dem Menschen eigen sei, scheint als Vorbild für Scrimas Idee einer „schöpferischen, antiphonischen und dialektischen Beziehung " zwischen Geist und Leib zu fungieren. Interessanter- und originellerweise kommt Scrima zur Idee der "großen Momente des Leibes“ zurück und betont noch einmal den übergreifenden Effekt des monastischen Lebens, als dasjenige Leben, das zur synthetischen, makrokosmischen und integrierenden Rolle des Menschen einen direkten Weg, ein sicheres Ziel bietet.

\section{Die Zeit}

Nach der Beziehung des menschlichen Wesens zur Materialität sollte auch die Beziehung zur Zeitlichkeit und Endlichkeit in den anthropologischen Gedanken Scrimas zur Diskussion kommen. Die Zeit wird als „eine charakteristische Dimension des menschlichen Wesens ${ }^{\text {“90 }}$ beschrieben. Sie ermöglicht das biologische und geistige Wachstum, das im Menschen als eine wesentliche Potenzialität eingeschrieben ist, sie markiert das Geschick des Seins als Geschöpf, beherbergt den Tod als „ontologische Modalität“, aber auch die Ereignisse einer höheren Ordnung, die den physischen Determinismus überschreiten und sich voll von einem besonderen Sinn für das Leben des Individuums erweisen. ${ }^{91}$

Der Mensch entdeckt „das Gesetz und das Wirken (lucrarea) der Zeit "92. Er blickt in das Geheimnis der Zeit hinein, welche durch besondere Umstände und Ereignisse die Geschöpfe der Welt umwandelt. In dieser Entdeckung ist auch eine Zurverfügungstellung der Zeit gegenüber dem Menschen inbegriffen. Man hat einen übergreifenden Blick über das Fließen der Zeit erhalten, und dies erlaubt, das „tiefe Werk des Menschen zu offen-

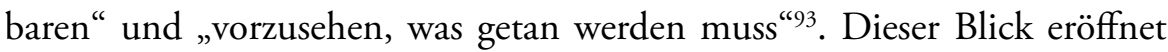
eine apokalyptische (offenbarende, enthüllende) aber auch prophetische Modalität des Sehens. Somit kommen die Menschen durch die Entdeckung

\footnotetext{
${ }^{87}$ Maximus Confessor, Ambiguum 7, PG 91, $1101 \mathrm{~B}$.

${ }^{88}$ Harper, „The Exemplar of Consubstantiality“, 103.

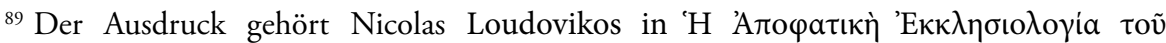

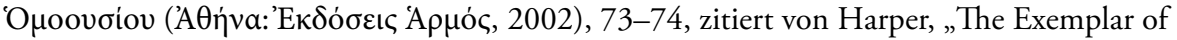
Consubstantiality", 103.

${ }^{90} A A, 208$.

${ }^{91}$ Ibidem.

${ }^{92}$ Ibidem.

${ }^{93}$ Ibidem.
} 
des Gesetzes der Zeit zur Verwirklichung ihrer Fähigkeit, „die Zeit zu schaffen, was auf wesentliche Weise eine göttliche Handlung ist “94. „Die Zeit schaffen" heißt, durch die Gnade Gottes zum apokalyptischen und prophetischen Blick über die Entwicklung, das Werden und die Umwandlung aller Dinge in der Zeit zu gelangen. Es heißt noch, in geistiger Erkenntnis die Wirkung der Zeit über die Geschöpfe zu verstehen, den Sinn der Ereignisse zu kennen. Der Mensch kommt also zur Ausübung einer in Essenz göttlichen Handlung und drückt dadurch die Göttlichkeit der Eikon aus, die er in sich hat. Scrima erläutert noch, dass die Entdeckung des Gesetzes der Zeit ein „kapitaler Akt für unsere geistige Erfüllung“ ist, und glaubt auch, dass die Vitae der Heiligen das Verfügen der Menschen über „das Maß dieser neuen, apokalyptischen Zeit" reichlich bezeugen. ${ }^{95}$

\section{Kontingenz, Freiheit und Mysterium}

Die Besprechung der Zeitlichkeit im Rahmen des Entwurfs einer apophatischen Anthropologie hat gezeigt, dass das menschliche Verständnis und Handeln in der Zeit dem "göttlichen Handeln“ ähneln kann. Dazu ist der Mensch über die Zufälligkeit erhoben, weil er Zugang zum Sinn des Geschehens im Fluss der Zeit haben kann. Wenn der Mensch auf diese Weise über die Zeit verfügen kann, stellt sich die Frage nach der Freiheit des Menschen und nach ihrer Beziehung zur Kontingenz. Laut Scrima können sich die apophatischen Kategorien des Mysteriums der Freiheit nicht entziehen. Er diskutiert die Freiheit keineswegs in einem moralischen oder ethischen Kontext, sondern als eine ontologische Kategorie:

Das geschaffene Wesen ist ein Mysterium der Kontingenz und Freiheit. Man kann dieses Mysterium nicht „kennen“ außer durch apophatische Kategorien: das ist das fundamental Gegebene der menschlichen ontologischen Erkenntnis (gnoză). ${ }^{96}$

Übrigens hat „Mysterium“ in Scrimas Diskurs immer eine ganz prägnante, integrative und offenbarende Funktion. In der apophatischen Anthropologie wird nicht das Mysterium vom Subjekt entziffert oder umfasst, sondern das Subjekt des Erkennens in das Mysterium umfasst und inbegriffen ${ }^{97}$.

\footnotetext{
${ }^{94}$ Ibidem.

${ }^{95}$ Ibidem.

${ }^{96} A A, 217$.

${ }^{97}$ Zum „Zusammenhang zwischen Apophatismus und Mysterium“, siehe Sibişan, Theanthropologie, 105. Vgl. Ideenjournal, in AA, 301: „Ein Mysterium kann nicht gedacht, vorgestellt (vorgeführt) oder bewiesen werden, denn dies würde bedeuten, dass wir es vergegenständlichen, was absurd ist: es kann nur durch eine Art konkreter Intuition / konkreten Erspürens erkannt werden."
} 
Zugang zum Mysterium zu haben, bedeutet nicht, aufzuhören im Mysterium zu sein, sondern gerade ins Mysterium einzutreten, und endlich ein persönliches Verhältnis mit ihm zu verwirklichen (a realiza în sfârşit raportul personal cu el)..$^{98}$

Die personale Beziehung mit dem Mysterium zu erleben ist schon eine Erfahrung des mystischen Lebens. Das Mystische wächst innerhalb des Mysteriums nicht wegen seiner Verborgenheit und Unzugänglichkeit oder wegen eines irrationalen Dranges, sondern durch eine persönliche Beziehung.

\section{Das Unendliche innerhalb des Endlichen}

Sowohl die Begriffe der Körperlichkeit und der Zeit als auch die Idee der Freiheit verlangen eine Auseinandersetzung mit den Gegensatzpaaren endlich/unendlich, begrenzt/unbegrenzt. Der Mensch scheint in seiner Leiblichkeit, in seiner Zeit, als erschaffenes und sterbliches Wesen, und in der Ausübung seiner Freiheit, im Bereich des Endlichen, angesiedelt zu sein. In diesem Zusammenhang beabsichtigt die apophatische Anthropologie zu zeigen, dass der Mensch Zugang zum Unendlichen und Teilhabe am Unbegrenzten hat, trotz seiner Ansiedlung im Bereich des Endlichen.

Die Ausarbeitung der Problematik des Unendlichen war immer eine Bestrebung in der Philosophie ${ }^{99}$ und in der Theologie ${ }^{100}$. Die Problematik der Endlichkeit, der Grenzen, der Andersheit zum Begrenztsein, ist auch für Scrimas Anthropologie wichtig. Scrima denkt ein Modell, wo das Unendliche organisch innerhalb des Endlichen auftauchen und transfigurierend wirken kann:

Das Unendliche ist „anders“ - allogenés - jenseits des Endlichen, wahrhaftig „an seinen Grenzen“ und nicht in seiner unbestimmten Ausdehnung, und deshalb kann es innerhalb des Endlichen

\footnotetext{
${ }^{98} A A$, S. 217.

${ }^{99}$ Emmanuel Levinas, „La philosophie et l'idée de l'Infini“, Revue de Métaphysique et de Morale 62, Nr. 3 (1957): 241-53; idem, Totalité et infini (La Haye: M. Nijhoff, 1980). Siehe dazu: Flora Bastiani, „Désacraliser l'infini. La place de la théologie dans la philosophie d'Emmanuel Levinas", Revue de théologie et de philosophie 61, Nr. 4 (2011): 335-44; Dan Arbib, Descartes, la métaphysique et l'infini (Paris: Presses Universitaires de France, 2017); Morgan Gaulin, Figures de l'infini. Du panthéisme de Schelling à Mallarmé (Bern: Peter Lang, 2017). ${ }^{100}$ Viele Studien sind in Bezug auf die Unendlichkeit bei Gregor von Nyssa erschienen, z. B.: David Bentley Hart, „The Mirror of the Infinite: Gregory of Nyssa in the Vestigia Trinitatis“, Modern Theology 18, Nr. 4 (2002): 541-61; Thomas Böhm, Theoria-Unendlichkeit-Aufstieg: philosophische Implikationen zu „De vita Moysis “von Gregor von Nyssa, Supplements to Vigiliae Christianae 35 (Leiden: Brill, 1996); Ekkehard Mühlenberg, Die Unendlichkeit Gottes bei Gregor von Nyssa. Gregors Kritik am Gottesbegriff der klassischen Metaphysik, Forschungen zur Kirchen- und Dogmengeschichte 16 (Göttingen: Vandenhoeck \& Ruprecht, 1966).
} 
erscheinen, als ein Prinzip der ontologischen Diskontinuität, als eine Eröffnung einer anderen Perspektive. ${ }^{101}$

Diese „Eröffnung einer anderen Perspektive“ durch ein Prinzip der „ontologischen Diskontinuität" erinnert an das unendliche Wirken des Heiligen Geistes - ein Wirken, das Gott gleichzeitig zeigt und verbirgt, unendlich offenbart und unendlich dem Begreifen entzieht ${ }^{102}$. Das Wirken des Heiligen Geistes, der das Unendliche im Endlichen auftauchen lässt, entspricht einer Dynamik, wo der Apophatismus zugleich sich selber bestätigt und transzendiert, auf spanungsvolle und paradoxe Weise ${ }^{103}$.

\section{Wandern und Gastfreundschaft}

Zum Schluss sind noch zwei Begriffe hinzuzufügen und zu untersuchen, die sowohl für Scrimas anthropologische Auffassung als auch für seine Darstellung der spirituellen Erfahrung zentral sind. Die Wanderung, d. h. der nomadische und itinerante Zustand, ist für A. Scrima die wesentliche Bestimmung des Lebens auf Erden des christlichen Menschen. In seinen Schriften, die im Band Die spirituelle Erfahrung und ihre Ausdrucksformen veröffentlicht worden sind, diskutiert Scrima den Gegensatz zwischen dem Sesshaften und dem Nomaden. Er setzt die Figuren von Kain und Abel als bildhaft für diesen Gegensatz ein. Für den Zustand der Wanderung wird nicht das Haus, sondern das Zelt das entscheidende Bild des Unterbringens: „Der Wandernde trägt, im Gegenteil, seine Wohnung mit sich. " ${ }^{104}$ Scrima hebt die Wichtigkeit des Zeltes in der biblischen Geschichte der Gastfreundschaft Abrahams im Hain von Mamre (Gen 18,1) und in der Erzählung der Verklärung Christi (Mk 9,5) hervor. ${ }^{105}$ Die Bezeichnung des

\footnotetext{
${ }^{101} A A, 223$.

${ }^{102}$ Siehe: Andrei Găitănaru, „The Double Resource of André Scrima’s Theology“, in André Scrima. Expérience spirituelle et langage théologique, 93-101, hier 100.

${ }^{103} A A, 77-78$ : Scrima spricht über den „Apophatismus, der den Apophatismus selber übersteigt".

${ }^{104}$ L'Expérience spirituelle et son langage. La tradition chrétienne [Experienţa spirituală şi limbajul său. Tradiţia creştină] im Band: Experienţa spirituală şi limbajele ei [Die spirituelle Erfahrung und ihre Ausdrucksformen], hg. von Anca Manolescu in Zusammenarbeit mit Radu Bercea, Vorwort von Anca Manolescu; übers. aus dem Französischen und Englischen und Anmerkungen von Anca Manolescu (Bukarest: Humanitas, 2008), 21-193, hier 185 (im Folgendem durch ES abgekürzt).

${ }^{105}$ Die Diskussion der Prinzipien oder Methoden der biblischen Hermeneutik André Scrimas kann nicht innerhalb dieser Analyse durchgeführt werden. Es sei hier nur angemerkt, dass die zwei Episoden parallel betrachtet werden, weil der Gegensatz Zelt/Haus als relevant für die ontologische Opposition zwischen dem Nomaden und dem Sedentären hervorgehoben wird. Es ist in der Kondition der Nomaden, dass der Mensch eine theophanische Erfahrung machen kann, dass er am Sich-Zeigen des Göttlichen teilhaben kann.
} 
Körpers als „Zelt“ in den paulinischen Briefen gewinnt laut Scrima eine wichtige anthropologische Bedeutung:

Der Körper selbst wird aus dieser Perspektive „Zelt" benannt, wie häufig der Heilige Paulus es tut. Das Zelt des Menschen par excellence ist das körperliche Zelt. Ich setzte mich nicht in den Körper hinein, um mich im Raum anzusiedeln, sondern, im Gegenteil, ich trage dieses Haus mit. ${ }^{106}$

Somit ist die Bestimmung des Menschen die eines Wanderers, sofern der Körper als „Zelt" betrachtet wird, das in der Reise oder Pilgerfahrt des hiesigen Lebens mitgetragen wird. Der völligen Annahme der Wanderschaft folgt unbedingt eine Entdeckung des totalen und radikalen Sinnes der Gastfreundschaft ${ }^{107}$. Es geht hier nicht um die gemütliche, partielle und temporäre Gastfreundschaft im Haus des sesshaften Menschen, sondern um die ganz offene, zur totalen Gemeinschaft einladende Gastfreundschaft des Nomaden.

Nur der Nomade kann die Einsicht davon haben, wie man die Gastfreundschaft bis zur letzten Grenze völlig ausüben kann. Das ist etwas ganz Anderes als die bloße Begegnung oder Unterbringung von jemandem [...]. Es gibt die Gemeinschaft und die Gegenseitigkeit der Gastfreundschaft; es handelt sich nicht um eine Geste in einer einzigen Richtung [...]. ${ }^{108}$

Der Nomade wird zu einer Figur der spirituellen Erfahrung; er ist gleichzeitig auch eine Darstellung einer anthropologischen Grundbestimmung des Menschen: das Unterwegssein zum Reich Gottes und zur Freude der Teilhabe am Unerschaffenen ${ }^{109}$. Diese Figur verortet den Menschen nicht im Horizont des Habens und des Konsumierens, sondern im reichen Gebiet der Tugenden wie Gerechtigkeit, Treue und Gastfreundschaft. Sie lässt einfach Raum für das Energetische, für das Schaffende und Schöpferische des Seins in einer unendlichen Bewegung, die der sabbatischen Berufung des Menschen nicht widerspricht, sondern sie antinomisch und apophatisch erhellt:

\footnotetext{
${ }^{106} E S, 185$.

${ }^{107}$ Zum Thema der Gastfreundschaft (ospitalitate) bei André Scrima, siehe: Manolescu, „André Scrima şi sensul metafizic al ospitalităţii“, in André Scrima - o gândire fără ţărmuri (Bukarest: Humanitas 2005), 35-54. Der Band veröffentlicht die Beiträge des Kolloquiums „Une pensée sans rivages. Ecuménisme et globalisation“, organisiert von New Europe College und vom Institut Ludwig Boltzmann in Bukarest am 24 Januar 2004.

${ }^{108}$ ES, 186.

${ }^{109}$ Zum Bild des Itinerars oder der Wanderung und zur Figur des Fremden, der gerade eine Sedentarität in sich selbst ausschließen und eine Art Familiarität mit Gott suchen will, siehe: Găitănaru, „The Double Resource“, 94.
} 
Die Wanderung (itineranţa) ist in den Horizont der Gerechtigkeit, der Treue, der Gastfreundschaft, der Energetik des Seins eingeschrieben; sie ist mit denjenigen assoziiert, die nicht sammeln und nicht lagern, um zu konsumieren. Wenn die Nomaden feiern, stellen sie alles sofort zur Verfügung. ${ }^{110}$

Nach Scrimas Auffassung entdeckt man den Sinn des Festes, der Gabe und des Teilens im Wanderleben der Nomaden wieder. Das Fest und die Gabe sowie die Gemeinschaft mit den anderen, in einem gemeinsamen Unterwegssein zu Gott, illustrieren die Bedeutung des Menschseins ${ }^{111}$.

\section{Anstelle von Schlussfolgerungen: Wenn Anfang und Ende sich treffen...}

Es ist keine einfache Aufgabe, eine Quintessenz von Scrimas Anthropologie darzustellen. Seine Sprache ist zugleich eine theozentrische, eine anthropologische und eine spirituell-mystische Sprache. Die Motive drehen sich in einem spiralförmigen Denken, das im Diskurs immer tiefere Ebenen des Apophatismus einfließen lässt. Die Negation, die Antinomie, das Bildhafte und das Jenseitige begegnen sich im Rahmen eines Apophatismus, der von der Theologie in die Anthropologie ausstrahlt und die Anthropologie zum Kern des Mysteriums des Menschen führt. Anfang und Ende des menschlichen Daseins treffen sich im Licht der Transfiguration des homo absconditus. In dieser Transfiguration des Menschen in Christus und durch den Heiligen Geist wird das Verborgene transparent und das Mysterium strahlend. ${ }^{112}$

Scrimas Anthropologie hat im Zentrum die Teilhabe des nach dem Bild Gottes geschaffenen Menschen an den ungeschaffenen Energien Gottes, am göttlichen Licht - diese ist für Scrima eine pneumatische Erfahrung, eine Erfahrung der Anwesenheit des Geistes. In der Vergöttlichung des Menschen begegnen sich die Präsenz Gottes in seinem Bild, die seit der Schöpfung des Menschen unverlierbar ist, mit der Präsenz der vergöttlichenden Gnade Gottes, im in Christus neu geschaffenen Menschen. Der anthropologische Apophatismus baut auf dem Mysterium der Beschaffenheit des Menschen ad imago dei auf und vertieft und vervollkommnet sich auf die Dimensionen

\footnotetext{
${ }^{110} E S, 188$.

${ }^{111}$ Für die wesentliche Identifikation „Mönch-Pilger“ und „die österliche Freude der Pilgerfahrt" (the pascal joy of pilgrimage) für Scrima und seinen biographischen Weg, siehe: Athanasios Giocas und Paul Ladouceur, „The Burning Bush Group and Father André Scrima in Romanian Spirituality“, Greek Orthodox Theological Review 52, Nr. 1-4 (2007): 37-61, hier 46.

${ }^{112} A A, 100, \mathrm{Vgl}$. Sibişan bezüglich des Mysteriums, Theanthropologie, 105: „Ihm sei weder Undurchsichtigkeit oder Opazität noch Obskurität zuzuschreiben.“
} 
des christologischen und pneumatologischen Apophatismus, die in der Vergöttlichung anwesend sind.

Einerseits kann die Anwesenheit Gottes im Menschen auch als Transfiguration oder als göttliche Assimilation verstanden werden. Andererseits ist die Vergöttlichung als Dynamik der göttlichen „Ikone“ im Menschen zu verstehen, wobei das Bild Gottes nicht in einer starren, positiv beschreibbaren Eigenschaft oder Wesensbestimmung besteht, sondern auf eine unbegreifliche und antinomische Reflexion des Mysteriums des Schöpfers, die im Geschöpf (also Menschen) stattfindet, hinweist. Diese Reflexion zieht den Menschen in ein unendliches Streben nach seinem Urbild, und macht ihn capax dei, fähig, Gott zu empfangen und zu erleiden. In dieser pathischen Beziehung erfährt das göttliche Bild eine immer größere Ähnlichkeit mit seinem Archetyp; die Vollkommenheit der Ähnlichkeit zeigt sich in der Neuschöpfung des Menschen in Jesus Christus, im neuen Adam und wahrhaften Protovater (engl. protoparent) der Menschheit. Christus ist der wirkliche Prototyp der Menschheit, weil die menschliche Natur in ihm die Permeabilität für Gott (also die maximale Fähigkeit zu pati Deum) wiedergewinnt. Diese Permeabilität dem Göttlichen gegenüber kann bei Scrima nicht nur in der Sprache der Teilhabe, sondern auch mithilfe des Vokabulars der „Mischung“ wiedergegeben werden, wobei A. Scrima von der traditionellen Vorsichtsmaßnahme des Modells der anthropologischen Perichorese Abstand nimmt und zum explanativen Potential des Bildes der „Mischung“ zurückkommt, ohne daraus stricto sensu einen theologischen Begriff zu entwickeln.

Die Erfüllung des Endziels des Menschen, das vom Moment der Schöpfung in seiner Beschaffenheit eingeschrieben wurde, ist zugleich eine Verwirklichung der sabbatischen Berufung des Menschen. In dieser Verwirklichung treffen sich die edenische Ruhe mit dem Frieden ohne Untergang des Reiches Gottes. Der Prozess dieser Verwirklichung beginnt im Herzen des Menschen, in der hesychia des Betens ohne Unterlass. In diesem Prozess, also im Horizont der durch den Heiligen Geist vollbrachten Transfiguration des Menschen, re-definiert sich das Verstehen der Körperlichkeit und der Beziehungen zwischen Anthropos und Kosmos: der Anthropos wird als Makrokosmos verstanden. Gleichzeitig erschließt sich ein anderes Verhältnis des Menschen zur Zeitlichkeit, wo der Mensch nicht (als unterdrücktes Wesen) „unter“ der Zeit steht, sondern auf göttliche Weise über die Zeit verfügt und zum Sinn der Ereignisse gelangt, der unter einem apokalyptischen Blick beleuchtet werden kann. Wenn dieses Verhältnis des Menschen zu Leiblichkeit, Zeitlichkeit und Endlichkeit schon in sich Antinomien enthält, muss umso mehr das Sich-Kreuzen von Kontingenz 


\section{Georgiana Huian}

und Freiheit im menschlichen Leben auf ein Mysterium hinweisen, welches die apophatische Methode braucht.

Letztendlich erlaubt die „feine Stickarbeit“ der apophatischen Denkund Sprachmuster ein Treffen mit der mystischen Erfahrung, wo Sich-Zeigen und Sich-Entziehen nicht nur komplementäre intellektuelle Kategorien sind, sondern ineinander verwobene lebendige Intuitionen des Absoluten. Das Wirken des Heiligen Geistes ist in dieser Gleichzeitigkeit von Zeigen und Verbergen, Offenbaren und Entziehen, und auch im Erscheinen des Unendlichen „an den Grenzen“ des Endlichen spürbar. Um für dieses Wirken, für das pneumatische Ereignis bereit zu sein, soll der Mensch auf der Erde im Zustand des Wanderns leben. Dazu soll er immer die Gastfreundschaft ausüben, als Treue zu seiner Berufung, die Jenseitigkeit und die göttliche Andersheit zu empfangen. Die Wanderung und die Gastfreundschaft werden also zu Grundbestimmungen des Unterwegsseins zum Reich Gottes zu topoi des spirituellen Itinerariums und der mystischen Umsiedelung. 